\title{
Peptide and Protein Quantification Using iTRAQ with Electron Transfer Dissociation
}

\author{
Doug Phanstiel, ${ }^{\mathrm{a}}$ Yi Zhang, ${ }^{\mathrm{c}}$ Jarrod A. Marto,, ${ }^{\mathrm{c}, \mathrm{d}}$ and Joshua J. Coon ${ }^{\mathrm{a}, \mathrm{b}}$ \\ ${ }^{a}$ Department of Chemistry, University of Wisconsin, Madison, Wisconsin, USA \\ ${ }^{b}$ Department of Biomolecular Chemistry, University of Wisconsin, Madison, Wisconsin, USA \\ 'Department of Cancer Biology and Blais Proteomics Center, Dana-Farber Cancer Institute, Boston, \\ Massachusetts, USA \\ d Department of Biological Chemistry and Molecular Pharmacology, Harvard Medical School, Boston, \\ Massachusetts, USA
}

Electron transfer dissociation (ETD) has become increasingly used in proteomic analyses due to its complementarity to collision-activated dissociation (CAD) and its ability to sequence peptides with post-translation modifications (PTMs). It was previously unknown, however, whether ETD would be compatible with a commonly employed quantification technique, isobaric tags for relative and absolute quantification (iTRAQ), since the fragmentation mechanisms and pathways of ETD differ significantly from CAD. We demonstrate here that ETD of iTRAQ labeled peptides produces $c$ - and $\dot{z}$-type fragment ions as well as reporter ions that are unique from those produced by CAD. Exact molecular formulas of product ions were determined by ETD fragmentation of iTRAQ-labeled synthetic peptides followed by high mass accuracy orbitrap mass analysis. These experiments revealed that ETD cleavage of the $N-C_{\alpha}$ bond of the iTRAQ tag results in fragment ions that could be used for quantification. Synthetic peptide work demonstrates that these fragment ions provide up to three channels of quantification and that the quality is similar to that provided by beam-type CAD. Protein standards were used to evaluate peptide and protein quantification of iTRAQ labeling in conjunction with ETD, beam-type CAD, and pulsed Q dissociation (PQD) on a hybrid ion trap-orbitrap mass spectrometer. For reporter ion intensities above a certain threshold all three strategies provided reliable peptide quantification (average error $<10 \%$ ). Approximately $36 \%$, $8 \%$, and $16 \%$ of scans identified fall below this threshold for ETD, HCD, and PQD, respectively. At the protein level, average errors were $2.3 \%, 1.7 \%$, and $3.6 \%$ for ETD, HCD, and PQD, respectively. (J Am Soc Mass Spectrom 2008, 19, 1255-1262) (C) 2008 American Society for Mass Spectrometry

$\mathrm{P}$ rotein quantification has become an important and, in many cases, critical component of modern mass spectrometry-based proteomic research [1-9]. Over the past decade, numerous quantification strategies have evolved-nearly all of them rely on the incorporation of stable isotopes for subsequent mass spectrometric sorting and relative quantification [1019]. Time and method of isotope integration distinguish these approaches. Whether introduced metabolically through heavy amino acids or chemically with differentially labeled tags at the peptide or protein level, mixing of the light and heavy (e.g., control and treated) peptides results in co-eluting peptide pairs with subtle, but measurably, different masses [10-12, 14-16, 20, 21].

In a clever departure from this paradigm, Pappin et al. described the concept of amine-reactive isobaric tagging [13]. Here, differentially isotopically labeled but isobaric amine-reactive tags (up to four) are embedded into peptides from as many as four separate peptide

Address reprint requests to Dr. J. J. Coon, Departments of Chemistry and Biomolecular Chemistry, University of Wisconsin, 1101 University Ave, Madison, WI 53706, USA. E-mail: jcoon@chem.wisc.edu pools (e.g., control and three treatment time points). Once labeled, the four samples are combined and peptides are sequenced individually by tandem mass spectrometry using collision-based dissociation methods [i.e., beam-type collision-activated dissociation (CAD) or pulsed Q dissociation (PQD)]. Identical peptides arising from each of the four samples co-elute and have equivalent $\mathrm{m} / \mathrm{z}$ values. During MS/MS, however, vibrational excitation induces cleavage of both the peptide backbone and the isobaric tag. Dissociation of the backbone gives rise to fragment ions characteristic of the peptide sequence; dissociation of the tag generates low mass product ions where each of the four labels creates a unique $m / z$ reporter peak. Because it allows for the simultaneous quantification of up to four samples, ITRAQ has become an important and powerful protein quantification methodology.

Due to the loss of low mass ions during resonant excitation (low mass cutoff), the use of iTRAQ labeling in conjunction with ion trap and ion trap hybrid mass spectrometers has been limited. That is, ion trap CAD often results in the inability to detect iTRAQ reporter ions due to the loss of low mass ions during precursor 
fragmentation [22]. However, the rapid scanning, excellent sensitivity, and ability to couple with other analyzers such as the orbitrap and Fourier transform ion cyclotron resonance (FT-ICR) have made ion traps among the most useful devices for protein and peptide identification [23]. Beam-type CAD is now available on hybrid ion trap-orbitrap mass spectrometers, but these systems only permit detection of product ions in the orbitrap mass analyzer, which is inherently slower and less sensitive that ion trap mass analysis [24]. PQD, a form of ion trap CAD designed to eliminate low mass cutoff, does allow for detection of low mass-to-charge fragment ions and is available on a some ion trap and ion trap hybrid mass spectrometers [25]. Griffin et al. have demonstrated that PQD is compatible with iTRAQ labeling and have characterized the quantitative merits of this approach [26, 27].

Electron transfer dissociation is complementary to $\mathrm{CAD}$ and can be especially useful for sequencing peptides containing post-translational modification (PTM) [28-36]. ETD allows for rapid peptide sequencing, with speeds similar to ion trap CAD, but is operated such that ions of mass lower than $100 \mathrm{~m} / \mathrm{z}$ are detected regardless of precursor $\mathrm{m} / \mathrm{z}$ (i.e., no low mass cut-off). Work by us and others demonstrate that ion trap CAD and ETD are complementary; however, ETD and other electron-based dissociation methods rely on free radicalinitiated peptide backbone cleavage and hence are not obviously compatible with the iTRAQ tagging strategy. Here we demonstrate that ETD produces iTRAQ reporter ions, unique from those produced by CAD, and that these reporters allow for peptide quantification of up to three different samples. Fragmentation of iTRAQ labeled peptides with ETD results in $c$ - and $\dot{z}$-type fragment ions and two fragment ions resulting from cleavage of the iTRAQ tag. One of the cleavages results in reporter ions that allow for quantitative comparison of up to three different samples. Synthetic peptides as well as digests of protein standards were used to evaluate the quality of iTRAQ based quantification in conjunction with ETD in an ETD enabled hybrid linear ion trap-orbitrap mass spectrometer. Peptide and protein quantification was compared using ETD, PQD, and beam-type CAD (HCD).

\section{Materials and Methods}

\section{Sample Preparation}

Synthetic peptides were obtained from the University of Wisconsin-Madison Biotechnology Center, standard proteins were purchased from Sigma-Adrich (St. Louis, $\mathrm{MO})$, and the iTRAQ labeling reagent was purchased from Applied Biosystems (Foster City, CA). Bovine serum albumin, $\beta$-casein, horse cytochrome $c$, $\beta$-lactoglobin, rabbit phosphorylase $\mathrm{B}$, and carbonic anhydrase were reduced, alkylated, and digested as previously described [37]. iTRAQ labeling was performed according to the manufacturer supplied protocol in $\sim 70 \%$ ethanol and $0.15 \mathrm{M}$ triethylammonium bicarbonate at room temperature for $1 \mathrm{~h}$. Samples were subsequently mixed, desalted using solid-phase extraction, dried to completion, and resuspended in $100 \mathrm{mM}$ acetic acid before LC-MS/MS analysis.

\section{Liquid Chromatography and Mass Spectrometry}

Synthetic peptides were resuspended in 30\% acetonitrile with $100 \mathrm{mM}$ acetic acid and infused in the mass spectrometer via static nanospray Econotips (New Objective, Woburn, MA). The six protein digest was separated on-line using nanoflow reversed-phase highperformance liquid chromatography (nRP-HPLC) as previously described [38]. Briefly, the sample was bomb-loaded onto a $5 \mathrm{~cm} \times 75 \mu \mathrm{m}$ i.d. precolumn packed with $5 \mu \mathrm{m} \mathrm{C18} \mathrm{reversed-phase} \mathrm{packing} \mathrm{material}$ (Alltech, Nicholasville, KY). This precolumn was then butt-connected to a $7 \mathrm{~cm} \times 50 \mu \mathrm{m}$ i.d. analytical column with Teflon tubing. The sample was eluted into the mass spectrometer using a $60 \mathrm{~min}$ linear gradient from $100 \mathrm{mM}$ acetic acid to $100 \mathrm{mM}$ acetic acid $70 \%$ acetonitrile at a flow rate of $\sim 60 \mathrm{~nL} / \mathrm{min}$.

ETD reactions and mass analysis were carried out in a hybrid linear ion trap-orbitrap mass spectrometer (Orbitrap; Thermo Fisher Scientific, Bremen, Germany), which was modified as previously described to perform ETD reactions [39]. A negative chemical ionization (NCI) source was fitted to the back of the instrument and connected to the back of the C-trap via a multipole. The linear ion trap was modified to enable charge-sign independent trapping (CSIT). Radical fluoranthene ions are generated in the NCI source and transported down the added multipole, through the C-trap and second multipole, and finally into the linear ion trap where ETD reactions proceed exactly as they would in a commercially available linear ion trap mass spectrometer. After fragmentation, product ions can either be analyzed by the linear ion trap or sent to the orbitrap mass analyzer for high mass accuracy detection. All ETD reactions were performed for $85 \mathrm{~ms}$. Precursor cation target values of 40,000 for ion trap mass analysis and 300,000 for orbitrap mass analysis were used. PQD and HCD collision energies were optimized on iTRAQ labeled synthetic peptides before LC-MS/MS runs. Normalized collision energies of 45 and 31 were used for HCD and PQD, respectively. Spectra from infused samples were averaged for 100 scans. LC-MS/MS experiments comprised of 10 scan events; an $\mathrm{MS}^{1}$ scan with orbitrap mass analysis followed by HCD (beamtype CAD in the collision cell followed by orbitrap mass analysis), PQD, and ETD of the three most abundant precursors. Fragment ions generated by PQD and ETD were analyzed in the ion trap mass analyzer while those produced by HCD were detected in the orbitrap mass analyzer. 


\section{Data Analysis}

$\mathrm{MS}^{2}$ spectra were searched using open mass spectrometry search algorithm (OMSSA) [40]. The database searched consisted of the six protein sequences for our standard peptides as well as a reversed human International Protein Index (IPI) database, which allowed false positive rate filtering [41, 42]. To limit false positive identifications, the results were filtered by precursor mass error and OMSSA e-value such that no reversed database entries were included. Software was written in-house to extract quantitative information from the .dta files. For each scan, peak intensities within $\pm 0.5 \mathrm{Da}$ (0.01 for HCD scans) of expected reporter ion $\mathrm{m} / \mathrm{z}$ ratios were summed. The software also grouped peptide identifications into protein identifications, calculated protein ratios by averaging peptide ratios, and provided standard deviation calculations for each protein. As shown in Figure 1, each channel was extremely pure so no corrections were made for isotopic purity.

Synthetic peptide impurities and unequal sample loss during preparation required normalization. To accomplish this, the synthetic peptide SSAAKAAAK was labeled with three iTRAQ tags (114, 115, and 116), mixed in 1:1:1 ratio, and infused into a hybrid linear ion trap-orbitrap mass spectrometer. The precursor population was then fragmented by beam-type CAD in the collision cell and analyzed in the orbitrap mass analyzer. The relative peak heights observed for the 114, 115 , and 116 peaks were used to normalize all subsequent ETD and HCD scans of labeled synthetic peptide mixtures. No normalization was performed on the quantitative data from LC-MS/MS analyses.

\section{Results}

\section{Fragmentation Pathways}

To determine the effect of iTRAQ labeling upon ETD fragmentation a synthetic peptide with the sequence HAAAHAAAH, no joke, was labeled with each of the iTRAQ tags (i.e., 114, 115, 116, and 117). Peptides from each group were separately ionized via nano electrospray (infusion) and sampled by an ETD-enabled linear ion trap-orbitrap hybrid mass spectrometer. Following the ETD reaction, the product ions were injected into the orbitrap for $\mathrm{m} / \mathrm{z}$ analysis. Orbitrap mass analysis revealed that numerous $c$ - and $\dot{z}$-type ions were generated. In this case, only the $\mathrm{N}$-terminus of the peptide contains an isobaric tag; thus, the entire $c$-type product ion series is increased by the exact mass of the intact isobaric tag $(144.1059,144.0996$, or 144.1021 Da depending on the tag used). Further, we find no cases wherein $c$-type ions have lost the isobaric tag.

A comparison of the low $\mathrm{m} / \mathrm{z}$ region produced following ETD tandem MS of unlabeled and labeled (117) precursors, however, reveals the presence of two new $\mathrm{m} / \mathrm{z}$ peaks: 104.1107 and 162.1354 (Figure 2). Note CAD cleavage of the peptide generates a reporter tag having a nominal $\mathrm{m} / \mathrm{z}$ value of 117 and this peak was not observed in the ETD spectrum. To identify the composition of the ETD-generated low $\mathrm{m} / \mathrm{z}$ peaks, we examined these mass spectral regions from the product ion spectra of each iTRAQ tagged peptide (e.g., 114, 115, 116 , and 117). Figure $1 \mathrm{a}-\mathrm{d}$ display the lower $\mathrm{m} / \mathrm{z}$ iTRAQ-specific ion from each of the iTRAQ tagged

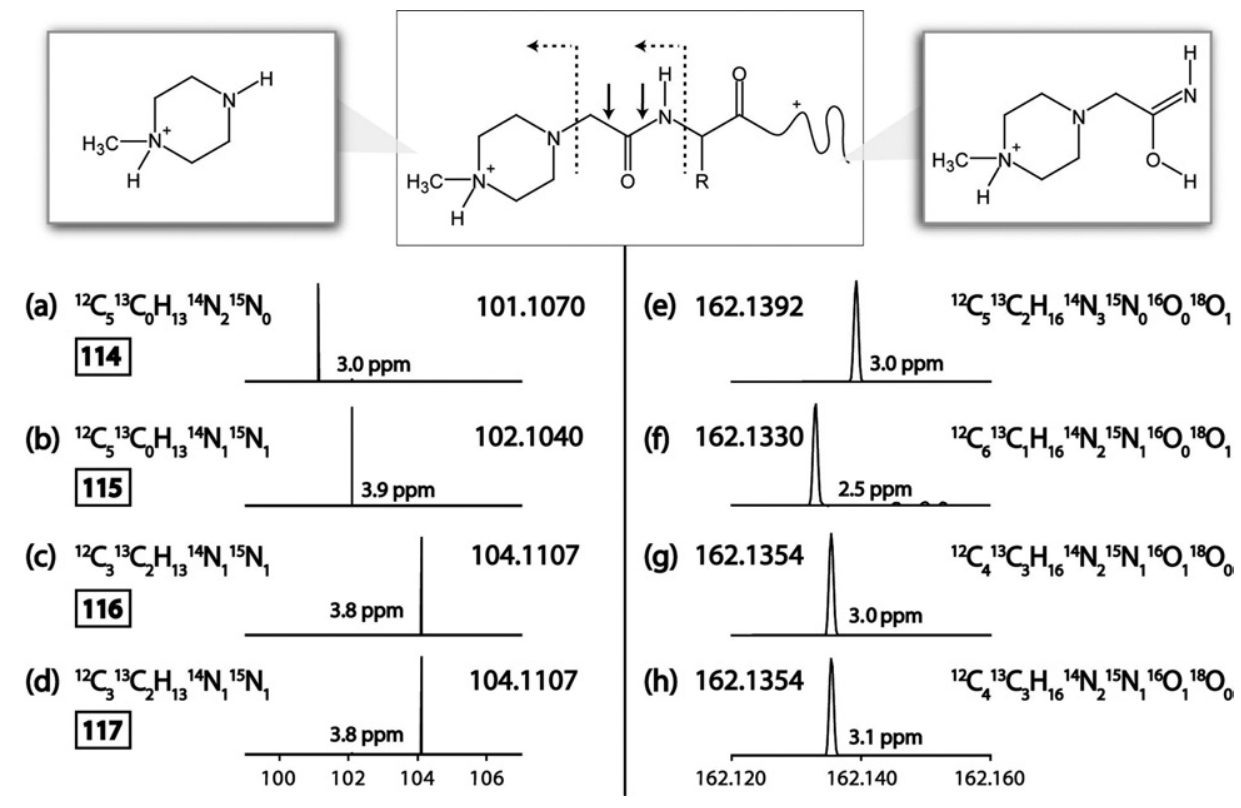

Figure 1. Proposed structures of ETD-generated iTRAQ reporter ions. The structure of an iTRAQ labeling reagent as attached to a peptide is shown (top middle). Bonds cleaved by CAD are indicated by solid arrows. Bonds cleaved by ETD are indicated by dotted arrows. Possible structures for cleavage products are shown in the top left and top right. (a)-(h) show mass spectrum of cleavage products generated by ETD of iTRAQ labeled peptides. Measured masses and error in ppm are shown. 


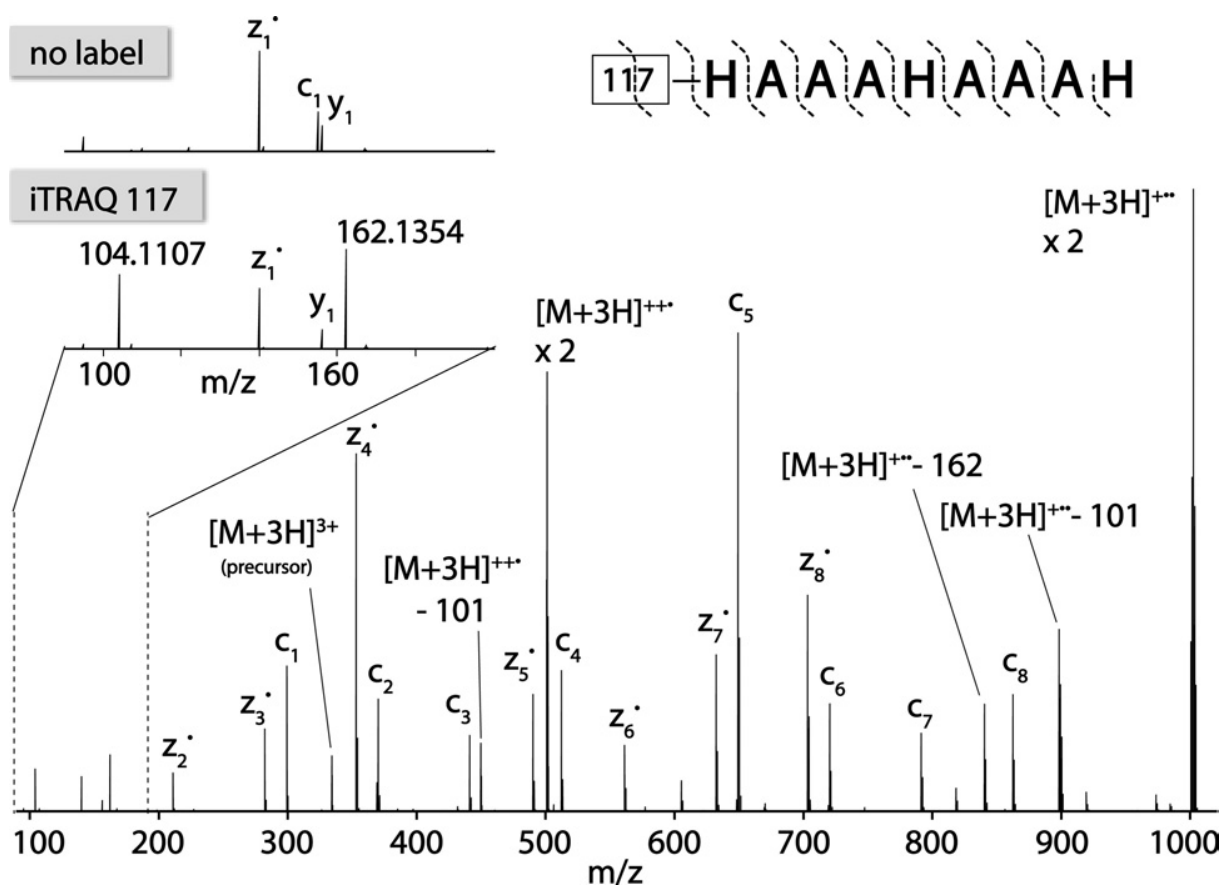

Figure 2. ETD MS/MS spectra with orbitrap mass analysis of synthetic peptide HAAAHAAAH labeled with the 117 iTRAQ tag. The pullout in the upper left depicts the low mass region observed upon ETD MS/MS of unlabeled HAAAAHAAAH. The lower frame is the same reaction but with HAAAAHAAAH labeled with the iTRAQ 117 tag.

species. From the accurate masses of these respective peaks we deduced the best-fit molecular formulas for each: 101.1073- ${ }^{12} \mathrm{C}_{5}{ }^{14} \mathrm{~N}_{2} \mathrm{H}_{13} ; 102.1044^{-12} \mathrm{C}_{5}{ }^{15} \mathrm{~N}_{1}{ }^{14} \mathrm{~N}_{1} \mathrm{H}_{13}$; 104.1111- ${ }^{13} \mathrm{C}_{2}{ }^{12} \mathrm{C}_{3}{ }^{15} \mathrm{~N}_{1}{ }^{14} \mathrm{~N}_{1} \mathrm{H}_{13}$. Although two molecular formulas were possible for the latter two of these masses within an error of $5 \mathrm{ppm}$, only one for each peak could be explained by the known structure of the iTRAQ tagging reagent. The theoretical $\mathrm{m} / \mathrm{z}$ values of each of these formulas fits the measured $\mathrm{m} / \mathrm{z}$ value to within $4 \mathrm{ppm}$ and is consistent with cleavage of the $\mathrm{N}$-methylpiperazine reporter region between the $\mathrm{N}-\mathrm{C}_{\alpha}$ of the iTRAQ tag. Note the intended CAD cleavage site of the reporter group is one methylene group downstream (i.e., between the carbonyl $\mathrm{C}$ and $\mathrm{C}_{\alpha}$ ). High mass accuracy measurements of the 162 peak were consistent with cleavage between the $\mathrm{N}-\mathrm{C}_{\alpha}$ of the first amino acid (Figure 1e-h).

From these data, we can identify where the heavy atoms are located in the iTRAQ tag. The $114 \mathrm{tag}$ contains two ${ }^{13} \mathrm{C}$ atoms (at the $\mathrm{C}_{\alpha}$ and the carbonyl) and an ${ }^{18} \mathrm{O} ; 115$ incorporates an ${ }^{15} \mathrm{~N}$ within the piperazine ring system, a ${ }^{13} \mathrm{C}$ at the $\mathrm{C}_{\alpha}$, and an ${ }^{18} \mathrm{O}$; 116 and 117 each have two ${ }^{13} \mathrm{C}$ atoms and one ${ }^{15} \mathrm{~N}$ within the piperazine ring system; they differ only in the placement of a third ${ }^{13} \mathrm{C}$ atom, 116 places it at the carbonyl $\mathrm{C}$ while 117 moves it to the $\mathrm{C}_{\alpha}$. Because ETD cleaves the $\mathrm{N}-\mathrm{C}_{\alpha}$ bond product ion spectra from the 116 and 117 tags generate identical reporter peaks, $104.1107 \mathrm{~m} / \mathrm{z}$. Therefore, only three unique reporter ions are available for relative quantification. Our results suggest slightly different chemical composi- tions from those originally reported by Pappin et al. [13]. However, our results agree with the chemical compositions provided in the manufacturer supplied reference guide.

\section{ETD-Generated Reporter Ions are Quantitative}

To determine the quantitative capability of these ETD-generated reporter ions, a synthetic peptide SSAAKAAAK was labeled with three different iTRAQ tags (114, 115, and 116). Samples were mixed in known ratios, infused into an ETD-enabled orbitrap mass spectrometer, fragmented by ETD, and analyzed using the linear ion trap mass analyzer. For comparison to beam-type CAD, each mixture was also fragmented by HCD with product ion $\mathrm{m} / \mathrm{z}$ detection performed in the orbitrap mass analyzer. Peptide ratios were calculated as previously described based on relative peaks areas of the reporter ions using the following formula; area (reporter ion of interest)/ (area \{all reporter ions\}) [26]. Thus all values fall between 0 and 1 with a 1:1:1 ratio $\sim 0.33$ for all three reporter ions. Relative peak areas of the 101,102, and 104 reporter ions corresponded well with peptide abundance for ratios ranging from 1:1 to 1:68. Figure 3 depicts observed versus expected percentage of reporter ion intensity. Least-squares fit yielded an equation of $y=0.8973 x+0.0342\left(R^{2}=0.99\right)$. This compares favorably with our beam-type CAD control; $y=0.9588 x+0.0137\left(R^{2}=0.99\right)$ (see Figure 3$)$. 


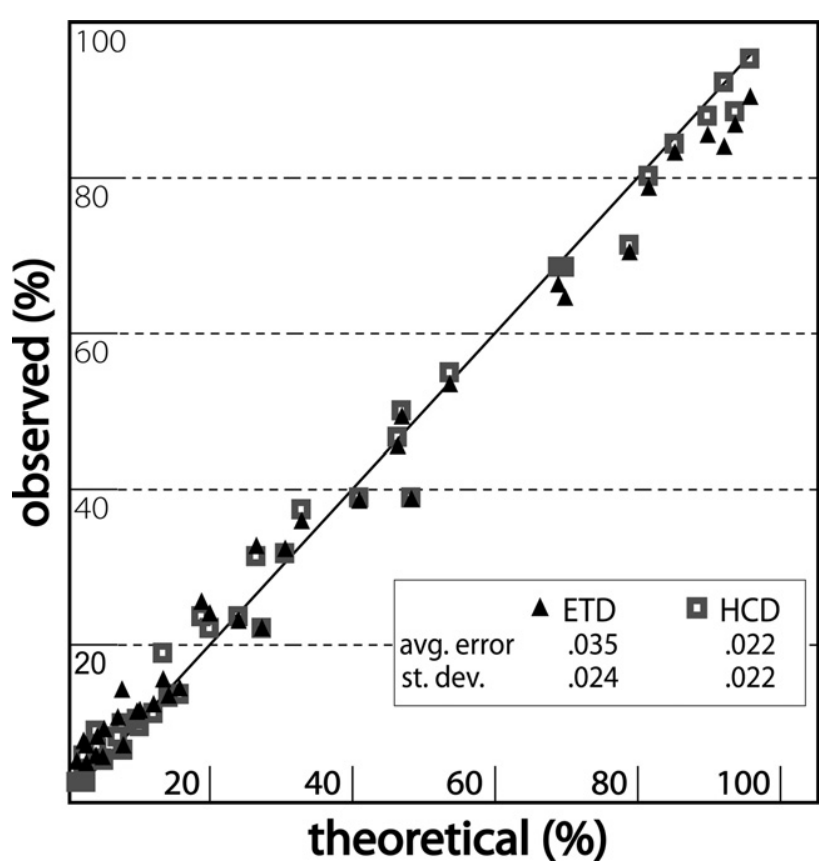

Figure 3. Theoretical versus observed ratios for both HCD and ETD fragmentation of an iTRAQ labeled peptide standard. Samples of labeled SSAAKAAAK were mixed in ratios ranging from 1:1 to 1:68. Ratios after ETD fragmentation and ion trap mass analysis are depicted as solid triangles. Ratios after beam-type CAD (HCD) fragmentation and orbitrap mass analysis are depicted as squares.

\section{Comparison of Protein Quantification Using ETD,} $P Q D$, and HCD

Quality of protein level quantification was determined using protein standards. Six protein standards were digested by trypsin, labeled with the same three iTRAQ labels $(114,115$, and 116), mixed in known ratios, and analyzed via LC-MS/MS with consecutive HCD, ETD, and PQD scans. Mixing ratios are BSA 1:1:1, carbonic anhydrase 1:0.5:0.1, $\beta$-lactoglobin 1:5:10, $\beta$-casein 1:2:4, phosphorylase B 1:0.5:0.2, and cytochrome $c$ 1:1:1. After peptide identification by database searching, average absolute error (absolute value of expected-observed) was compared with the sum total intensity of iTRAQ reporter ions in that scan. Figure 4 shows the number of scans that produced confident peptide identifications as a function of sum total reporter ion intensity. Scans that produced confident peptide identifications were binned according to the sum of all three reporter ion intensities. The bar graphs indicate how many scans fall into each bin while the line graphs above indicate the average quantitative error. For all scan types, average error decreased as reporter intensity increased. For ion trap scans (ETD and PQD), scans that exhibited reporter ion intensities summing to less than 1000 exhibited poor quantification; absolute error $=$ $26 \%$ for ETD, and $14 \%$ for PQD. For HCD scans with orbitrap detection scans with reporter ion intensities summing to less than 100,000 resulted in an error of $21 \%$. However, above these values all three scan types resulted in average errors of less than $10 \%$. Thus, each mass analyzer appears to have a threshold intensity for reporter ions below which quantitative results are unreliable.

Interestingly, $\sim 36 \%$ of all ETD scans that produced a confident identification exhibited low reporter ion intensities compared with only $8 \%$ of HCD scans and $16 \%$

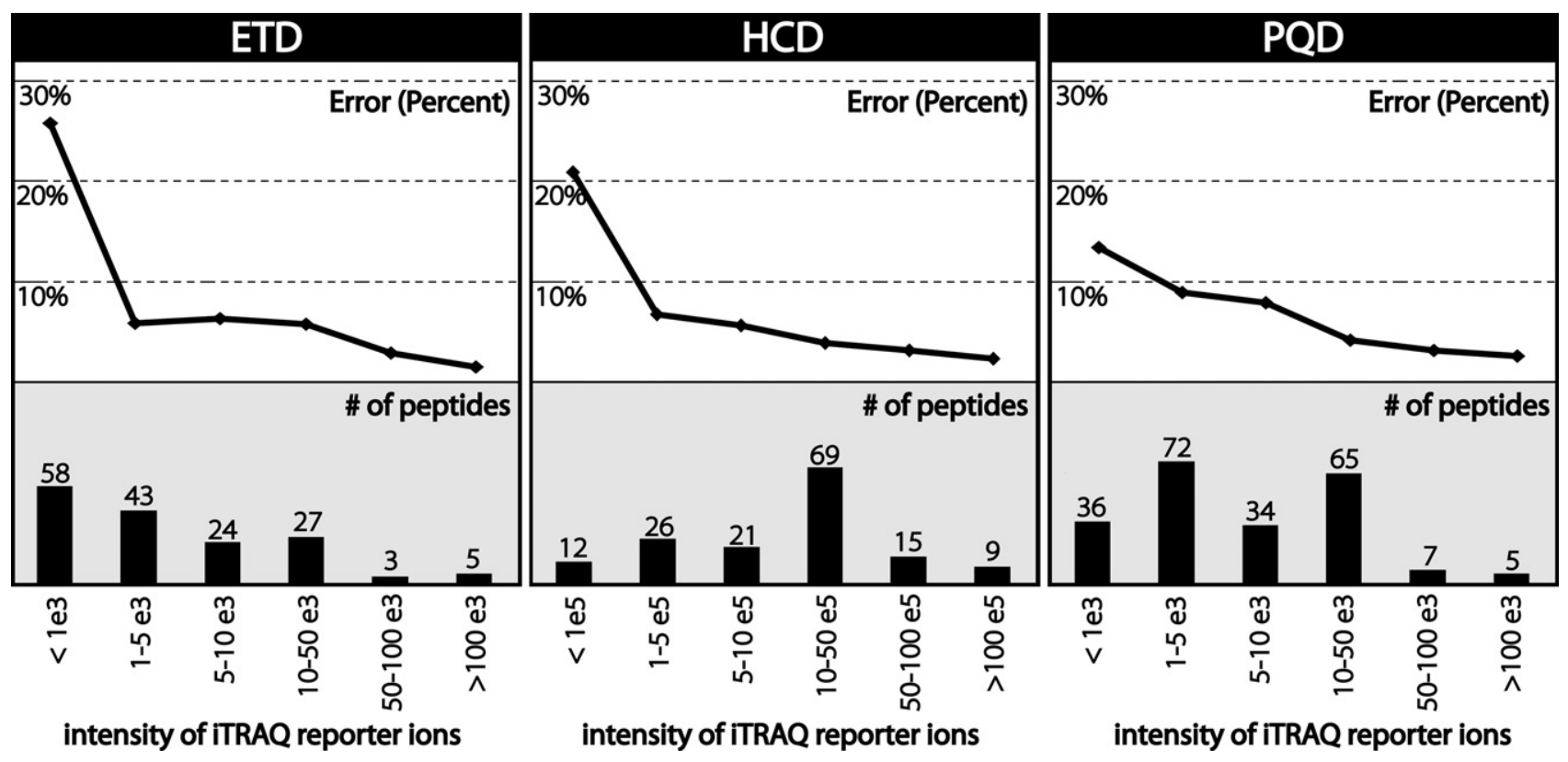

Figure 4. Quantitative error as a function of total reporter ion intensity. Scans were binned according to the sum of all three reporter ion intensities. Bar graphs depict the number of scans that fall into each bin. The line graph above depicts the average error for each bin. All three fragmentation methods exhibit high error $(>10 \%)$ below a certain intensity threshold; however, ETD generated a large number of scans that fall below this threshold. 


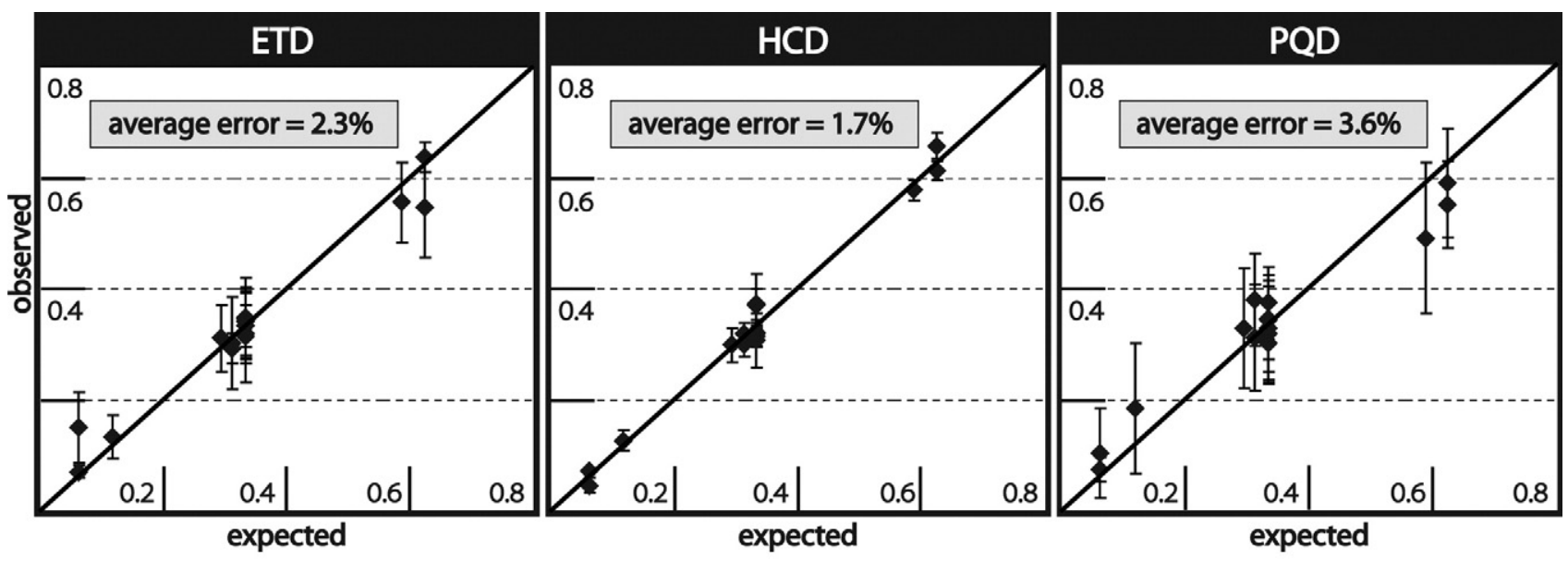

\begin{tabular}{|lccccccc|} 
& \multicolumn{3}{c}{ Mixing ratios } & & \multicolumn{3}{c}{ Peptides } \\
\cline { 2 - 4 } \cline { 6 - 8 } Protein & $\mathbf{1 1 4}$ & $\mathbf{1 1 5}$ & $\mathbf{1 1 6}$ & & ETD & HCD & PQD \\
\hline BSA & 1 & 1 & 1 & 31 & 35 & 62 \\
Cytochrome C & 1 & 1 & 1 & 17 & 5 & 20 \\
Beta-Casein & 1 & 2 & 4 & & 0 & 0 & 0 \\
Beta-lactoglobin & 1 & 5 & 10 & 28 & 41 & 59 \\
Phosphorylase B & 1 & 0.5 & 0.2 & 22 & 6 & 31 \\
Carbonic Anhydrase & 1 & 0.5 & 0.1 & & 4 & 6 & 11 \\
\hline
\end{tabular}

Figure 5. Protein level quantification using iTRAQ labeling in conjunction with ETD, PQD, and HCD. (Top) Each diamond denotes the ratio of one iTRAQ channel averaged across all identified peptides corresponding to one protein. Error bars indicate standard deviation. (Bottom) The table describes the ratios in which the proteins were mixed as well as the number of peptides used to quantify each protein. Note, although $\beta$-casein was identified by all fragmentation techniques, none of the peptides exhibited reporter ion ratios above the minimum threshold required for accurate quantification.

of PQD scans. One possible explanation for the high proportion of scans that exhibit low intensity reporter ion populations is that ETD does not result in preferential cleavage of the $\mathrm{N}-\mathrm{C}_{\alpha}$ bond of the iTRAQ tag. CAD is known to preferentially cleave the weakest bonds in the peptide backbone. iTRAQ tagging reagents were designed specifically so that CAD would preferentially cleave this bond, producing very intense reporter ion peaks. ETD cleaves randomly along the peptide backbone and is largely unaffected by amino acid composition and/or chemical modification. Therefore, ETD does not always produce intense iTRAQ reporter ions.

For the evaluation of protein quantification, only scans with sum total reporter ion intensities of 1000 or greater were included for ion trap measurements, and 100,000 for spectra acquired in the orbitrap. The observed ratios were averaged across all scans for each of the proteins identified. Figure 5 depicts expected versus observed ratios with standard deviations shown as error bars. The average difference between expected and observed was similar for all three dissociation methods $(\mathrm{ETD}=2.2 \%, \mathrm{PQD}=3.6 \%$, and $\mathrm{HCD}=1.7 \%)$. This compares favorably with the $<6 \%$ error originally reported for protein level quantification using iTRAQ labeling and beam-type CAD fragmentation [13].
ETD is limited by poor fragmentation efficiency of doubly charged peptides as well as peptides with high amino acid-to-charge ratios [30,37]. These caveats can be overcome by the use of supplemental activation of the nondissociated electron transfer products [34, 37]. However, supplemental activation, much like CAD, results in the loss of low mass ions and is therefore incompatible with iTRAQ quantification. More tractable approaches include the use different enzymes (e.g., Lys-C) or modified digestion conditions (incomplete tryptic digests) that result in more highly charged precursors. Chemical approaches may also be used to increase precursor charge states. Recently, Kjeldsen et al. have demonstrated that addition of $m$-nitrobenzyl alcohol to the liquid chromatography mobile phase increases the average charge states of precursors [32]. The more highly charged precursors exhibited increased ETD fragmentation efficiency. Introduction of basic moieties to the peptide through chemical modification before mass spectrometry may also provide enhancement of ETD fragmentation efficiency, and though we have not yet measured it in depth, the addition of the basic iTRAQ group appears to broadly elevate peptide charge. This could provide an added benefit for ETD fragmentation, and is a subject of current investigation in our laboratory. 


\section{Conclusions}

We have demonstrated that ETD of iTRAQ labeled peptides produces $c$ - and $\dot{z}$-type fragment ions and generates unique reporter ions that allow for peptide and protein quantification. Since the 116 and 117 tags produce the same reporter ion after fragmentation by ETD, the user is limited to only three channels of relative quantification. Synthetic peptides were used to evaluate the quality of quantification provided by ETD fragmentation of iTRAQ labeled peptides, and the results are comparable with those published using beamtype CAD as well as with our own beam-type CAD controls. Furthermore, we have compared protein quantification using iTRAQ and three different fragmentation strategies. All strategies provided reliable quantitative information when reporter ions exceeded a certain intensity threshold. However, compared with HCD and PQD, a high percentage $(\sim 36 \%)$ of the scans identified by ETD exhibited low reporter ion intensities. Taking efforts to increase precursor charge states may help reduce the percentages of identified peptide that exhibit low intensity iTRAQ reporter ions.

A major advantage of this approach is that it enables multiplexed quantification to be performed on all mass spectrometers capable of ETD, regardless of their ability to perform PQD or beam-type CAD. Furthermore, iTRAQ labeling can now be used in conjunction with ETD for the quantitative analysis of phosphorylated peptides and other post-translationally modified peptides that can be difficult to sequence by CAD. Lastly, decision tree based mass spectrometry approaches have recently been shown to enhance peptide and protein identification [43]. By combining iTRAQ labeling with ETD and either PQD or beam-type CAD, it may be possible to maximize peptide identifications while retaining the ability to perform multiplexed quantification.

A version of iTRAQ has recently been released that allows for up to eight channels of quantification. Since the reporter ion structure remains the same, it seems likely that ETD will be compatible with these reagents. Further experiments should confirm this supposition and determine how many channels of quantification it can provide.

\section{Acknowledgments}

The authors thank Graeme McAlister for instrument support. They acknowledge financial support for this work by the University of Wisconsin-Madison, Thermo Scientific, the Beckman Foundation, the American Society of Mass Spectrometry, Eli Lilly, the National Science Foundation (0701846; 0747990 both to J.J.C.), and the NIH (1R01GM080148 to J.J.C.). D.P. gratefully acknowledges support from NIH predoctoral fellowship (the Genomic Sciences Training Program, NIH 5T32HG002760).

\section{References}

1. Ong, S. E.; Foster, L. J.; Mann, M. Mass Spectrometric-Based Approaches in Quantitative Proteomics. Methods 2003, 29, 124-130.
2. Bantscheff, M.; Schirle, M.; Sweetman, G.; Rick, J.; Kuster, B. Quantitative Mass Spectrometry in Proteomics: A Critical Review. Anal. Bioanal. Chem. 2007, 389, 1017-1031.

3. Aebersold, R.; Mann, M. Mass Spectrometry-Based Proteomics. Nature 2003, 422, 198-207.

4. Heck, A. J. R.; Krijgsveld, J. Mass Spectrometry-Based Quantitative Proteomics. Expert Rev. Proteom. 2004, 1, 317-326.

5. Righetti, P. G.; Campostrini, N.; Pascali, J.; Hamdan, M.; Astner, H. Quantitative Proteomics: A Review of Different Methodologies. Eur. J. Mass Spectrom. 2004, 10, 335-348.

6. Ong, S. E.; Mann, M. Mass Spectrometry-Based Proteomics Turns Quantitative. Nat. Chem. Biol. 2005, 1, 252-262.

7. Julka, S.; Regnier, F. Quantification in Proteomics Through Stable Isotope Coding: A Review. J. Proteome Res. 2004, 3, 350-363.

8. Leitner, A.; Lindner, W. Chemistry Meets Proteomics: The Use of Chemical Tagging Reactions for MS-Based Proteomics. Proteomics 2006, 6, 5418-5434.

9. Conrads, T. P.; Issaq, H. J.; Veenstra, T. D. New Tools for Quantitative Phosphoproteome Analysis. Biochem. Biophys. Res. Commun. 2002, 290, 885-890.

10. Ong, S. E.; Blagoev, B.; Kratchmarova, I.; Kristensen, D. B.; Steen, H. Pandey, A.; Mann, M. Stable Isotope Labeling by Amino Acids in Cell Culture, SILAC, as a Simple and Accurate Approach to Expression Proteomics. Mol. Cell. Proteom. 2002, 1, 376-386.

11. Gygi, S. P.; Rist, B.; Gerber, S. A.; Turecek, F.; Gelb, M. H.; Aebersold, R. Quantitative Analysis of Complex Protein Mixtures Using IsotopeCoded Affinity Tags. Nat. Biotechnol. 1999, 17, 994-999.

12. Oda, Y.; Huang, K.; Cross, F. R.; Cowburn, D.; Chait, B. T. Accurate Quantitation of Protein Expression and Site-Specific Phosphorylation. Proc. Nat. Acad. Sci. U.S.A. 1999, 96, 6591-6596.

13. Ross, P. L.; Huang, Y. L. N.; Marchese, J. N.; Williamson, B.; Parker, K.; Hattan, S.; Khainovski, N.; Pillai, S.; Dey, S.; Daniels, S.; Purkayastha, S.; Juhasz, P.; Martin, S.; Bartlet-Jones, M.; He, F.; Jacobson, A.; Pappin, D. J. Multiplexed Protein Quantitation in Saccharomyces cerevisiae using amine-reactive isobaric tagging reagents. Mol. Cell. Proteom. 2004, 3, $1154-1169$.

14. Goshe, M. B.; Conrads, T. P.; Panisko, E. A.; Angell, N. H.; Veenstra T. D.; Smith, R. D. Phosphoprotein Isotope-Coded Affinity Tag Approach for Isolating and Quantitating Phosphopeptides in ProteomeWide Analyses. Anal. Chem. 2001, 73, 2578-2586.

15. Mirgorodskaya, O. A.; Kozmin, Y. P.; Titov, M. I.; Korner, R.; Sonksen C. P.; Roepstorff, P. Quantitation of Peptides and Proteins by MatrixAssisted Laser Desorption/Ionization Mass Spectrometry Using O-18Labeled Internal Standards. Rapid Commun. Mass Spectrom. 2000, 14, $1226-1232$.

16. Yao, X. D.; Freas, A.; Ramirez, J.; Demirev, P. A.; Fenselau, C. Proteolytic O-18 Labeling for Comparative Proteomics: Model Studies with Two Serotypes of Adenovirus. Anal. Chem. 2001, 73, 2836-2842.

17. Pasa-Tolic, L.; Jensen, P. K.; Anderson, G. A.; Lipton, M. S.; Peden, K. K.; Martinovic, S.; Tolic, N.; Bruce, J. E.; Smith, R. D. High Throughput Proteome-Wide Precision Measurements of Protein Expression Using Mass Spectrometry. J. Am. Chem. Soc. 1999, 121, 7949-7950.

18. Ishihama, Y.; Oda, Y.; Tabata, T.; Sato, T.; Nagasu, T.; Rappsilber, J.; Mann, M. Exponentially Modified Protein Abundance Index (emPAI) for Estimation of Absolute Protein Amount in Proteomics by the Number of Sequenced Peptides Per Protein. Mol. Cell. Proteom. 2005, 4 1265-1272.

19. Zhou, H. L.; Ranish, J. A.; Watts, J. D.; Aebersold, R. Quantitative Proteome Analysis by Solid-Phase Isotope Tagging and Mass Spectrometry. Nat. Biotechnol. 2002, 20, 512-515.

20. Faca, V.; Coram, M.; Phanstiel, D.; Glukhova, V.; Zhang, Q.; Fitzgibbon, M.; McIntosh, M.; Hanash, S. Quantitative Analysis of Acrylamide Labeled Serum Proteins by LC-MS/MS. J. Proteome Res. 2006, 5, 2009 2018.

21. Wu, C. C.; MacCoss, M. J.; Howell, K. E.; Matthews, D. E.; Yates, J. R Metabolic Labeling of Mammalian Organisms with Stable Isotopes for Quantitative Proteomic Analysis. Anal. Chem. 2004, 76, 4951-4959.

22. Schwartz, J. C.; Senko, M. W.; Syka, J. E. P. A Two-Dimensional Quadrupole Ion Trap Mass Spectrometer. J. Am. Soc. Mass Spectrom. 2002, 13, 659-669.

23. Domon, B.; Aebersold, R. Review-Mass Spectrometry and Protein Analysis. Science 2006, 312, 212-217.

24. Olsen, J. V.; Macek, B.; Lange, O.; Makarov, A.; Horning, S.; Mann, M. Higher-Energy C-Trap Dissociation for Peptide Modification Analysis. Nature Methods 2007, 4, 709-712.

25. Schwartz, J. C., Syka, J. P., Quarmby, S. T. Improving the Fundamentals of MSN on 2D Ion Traps: New Ion Activation and Isolation Techniques. Proceedings of the 53rd ASMS Conference on Mass Spectrometry; San Antonio, TX, June 2005.

26. Griffin, T. J.; Xie, H. W.; Bandhakavi, S.; Popko, J.; Mohan, A.; Carlis, J. V.; Higgins, L. iTRAQ Reagent-Based Quantitative Proteomic Analysis on a Linear Ion Trap Mass Spectrometer. J Proteome Res. 2007, 6, 4200-4209.

27. Meany, D. L.; Xie, H. W.; Thompson, L. V.; Arriaga, E. A.; Griffin, T. J. Identification of Carbonylated Proteins from Enriched Rat Skeletal Muscle Mitochondria Using Affinity Chromatography-Stable Isotope Labeling and Tandem Mass Spectrometry. Proteomics 2007, 7, 11501163.

28. Chi, A.; Huttenhower, C.; Geer, L. Y.; Coon, J. J.; Syka, J. E. P.; Bai, D. L.; Shabanowitz, J.; Burke, D. J.; Troyanskaya, O. G.; Hunt, D. F. Analysis 
of Phosphorylation Sites on Proteins from Saccharomyces cerevisiae by Electron Transfer Dissociation (ETD) Mass Spectrometry. Proc. Nat. Acad. Sci. U.S.A. 2007, 104, 2193-2198.

29. Syka, J. E. P.; Coon, J. J.; Schroeder, M. J.; Shabanowitz, J.; Hunt, D. F. Peptide and Protein Sequence Analysis by Electron Transfer Dissociation Mass Spectrometry. Proc. Nat. Acad. Sci. U.S.A. 2004, 101, 95289533.

30. Good, D. M.; Wirtala, M.; McAlister, G. C.; Coon, J. J. Performance Characteristics of Electron Transfer Dissociation Mass Spectrometry. Mol. Cell. Proteom. 2007, 6, 1942-1951.

31. Molina, H.; Horn, D. M.; Tang, N.; Mathivanan, S.; Pandey, A. Global Proteomic Profiling of Phosphopeptides Using Electron Transfer Dissociation Tandem Mass Spectrometry. Proc. Nat. Acad. Sci. U.S.A. 2007, 104, 2199-2204

32. Kjeldsen, F.; Giessing, A. M. B.; Ingrell, C. R.; Jensen, O. N. Peptide Sequencing and Characterization of Post-Translational Modifications by Enhanced Ion-Charging and Liquid Chromatography Electron-Transfer Dissociation Tandem Mass Spectrometry. Anal. Chem. 2007, 79, 9243 9252.

33. Mikesh, L. M.; Ueberheide, B.; Chi, A.; Coon, J. J.; Syka, J. E. P.; Shabanowitz, J: Hunt, D. F. The Utility of ETD Mass Spectrometry in Proteomic Analysis. Biochim. Biophys. Acta Prot. Proteom. 2006, 1764, 1811-1822.

34. Wu, S. L.; Huehmer, A. F. R.; Hao, Z. Q.; Karger, B. L. On-Line LC-MS Approach Combining Collision-Induced Dissociation (CID), ElectronTransfer Dissociation (ETD), and CID of an Isolated Charge-Reduced Species for the Trace-Level Characterization of Proteins with PostTranslational Modifications. J. Proteome Res. 2007, 6, 4230-4244.

35. Gunawardena, H. P.; Emory, J. F.; McLuckey, S. A. Phosphopeptide Anion Characterization via Sequential Charge Inversion and ElectronTransfer Dissociation. Anal. Chem. 2006, 78, 3788-3793.
36. Hogan, J. M.; Pitteri, S. J.; Chrisman, P. A.; McLuckey, S. A. Complementary Structural Information from a Tryptic N-Linked Glycopeptide via Electron Transfer Ion/Ion Reactions and Collision-Induced Dissociation. J. Proteome Res. 2005, 4, 628-632.

37. Swaney, D. L.; McAlister, G. C.; Wirtala, M.; Schwartz, J. C.; Syka, J. E. P.; Coon, J. J. Supplemental Activation Method for High-Efficiency Electron-Transfer Dissociation of Doubly Protonated Peptide Precursors. Anal. Chem. 2007, 79, 477-485.

38. McAlister, G. C.; Phanstiel, D.; Good, D. M.; Berggren, W. T.; Coon, J. J Implementation of Electron-Transfer Dissociation on a Hybrid Linear Ion Trap Orbitrap Mass Spectrometer. Anal. Chem. 2007, 79, 3525-3534.

39. McAlister, G. C.; Berggren, W. T.; Horning, S., Makarov, A.; Phanstiel, D.; Griep-Raming, J.; Stafford, G.; Swaney, D. L.; Syka, J. E. P.; Zabrouskov, V.; Coon, J. J. A Proteomics Grade Electron Transfer Dissociation-Enabled Hybrid Linear Ion Trap Orbitrap Mass Spectrometer. J. Proteome Res. (in press).

40. Geer, L. Y.; Markey, S. P.; Kowalak, J. A.; Wagner, L.; Xu, M.; Maynard, D. M.; Yang, X. Y.; Shi, W. Y.; Bryant, S. H. Open Mass Spectrometry Search Algorithm. J Proteome Res. 2004, 3, 958-964.

41. Peng, J. M.; Elias, J. E.; Thoreen, C. C.; Licklider, L. J.; Gygi, S. P. Evaluation of Multidimensional Chromatography Coupled with Tandem Mass Spectrometry (LC/LC-MS/MS) for Large-Scale Protein Analysis: The Yeast Proteome. J. Proteome Res. 2003, 2, 43-50.

42. Elias, J. E.; Gygi, S. P. Target-Decoy Search Strategy for Increased Confidence in Large-Scale Protein Identifications by Mass Spectrometry. Nature Methods 2007, 4, 207-214.

43. McAlister, G. C.; Swaney, D.; Griep-Raming, J.; Makarov, A.; Lange, O.; Schwartz, J.C.; Syka, J. E. P.; Horning, S.; Stafford, G.; Coon, J. J. A Probabilistic Decision Tree-Driven Tandem Mass Spectrometer for Shotgun Proteomics. (submitted). 